\title{
Comparison of fixed- and mobile-bearing total knee arthroplasty with a mean five-year follow-up: A meta-analysis
}

\author{
MENGQI CHENG $^{1 *}$, DESHENG CHEN $^{2 *}$, YONGYUAN GUO ${ }^{1}$, CHEN ZHU $^{1}$ and XIANLONG ZHANG ${ }^{1}$ \\ ${ }^{1}$ Department of Orthopedics, The Sixth Affiliated People's Hospital, Medical School of Shanghai Jiaotong University, \\ Shanghai 200233; ${ }^{2}$ Department of Orthopedics, The General Hospital of Ningxia Medical University, \\ Yinchuan, Ningxia 750004, P.R. China
}

Received February 1, 2013; Accepted April 23, 2013

DOI: $10.3892 / \mathrm{etm} .2013 .1122$

\begin{abstract}
Controversy exists regarding the clinical and radiological differences in outcomes between fixed-bearing (FB) and mobile-bearing (MB) total knee arthroplasties (TKAs) at the mid- or long-term follow-up. We therefore conducted a meta-analysis and systematic review of randomized controlled trials (RCTs) that have evaluated FB and MB TKAs. We searched the Cochrane Central Register of Controlled Trials (CENTRAL) in the Cochrane Library, Medline and Embase. The data, including demographic information, methodological quality, duration of follow-up, clinical and radiographical outcomes, patient preferences and complications, were extracted. The methodological quality of the studies was assessed in accordance with the guidelines presented in the Cochrane Handbook for Systematic Reviews of Interventions. Nine trials, studying 1,821 knees, were eligible for data extraction and meta-analysis. The Knee Society score and the maximum knee flexion demonstrated no difference between the FB and MB groups ( $\mathrm{P}=0.47$ and $\mathrm{P}=0.72$, respectively). Similarly, no difference was revealed between the groups for radiological outcomes or general health results. An increased number of high-quality RCTs with long-term follow-ups are required to validate the results.
\end{abstract}

\section{Introduction}

Total knee arthroplasty (TKA) has become a successful procedure for treating end-stage osteoarthritis and rheumatoid arthritis (1-5). The mobile-bearing (MB) TKA design is assumed to provide a greater freedom of motion compared with

Correspondence to: Professor Xianlong Zhang, Department of Orthopedics, The Sixth Affiliated People's Hospital, Medical School of Shanghai Jiaotong University, 600 Yishan Road, Shanghai 200233, P.R. China

E-mail: orthopedics2012@163.com

*Contributed equally

Key words: total knee arthroplasty, fixed-bearing, mobile-bearing, meta-analysis the fixed-bearing $(\mathrm{FB})$ variant, since the insert does not restrict the natural movements of the femoral component. This enables the reproduction of tibial internal rotation during flexion, reduces contact stresses and linear wear of the polyethylene, and improves patellofemoral tracking (6-10). This may, in turn, have a positive effect on the fixation of the prosthesis to the bone, and thereby reduce the risk of a loosening of the implant occurring.

Numerous trials have focused on the comparison of clinical outcomes between MB and FB TKAs. During the short-term follow-up period, no significant difference in clinical improvement has been demonstrated between MB- and FB-implant groups (11-16). In a prospective study, Hanusch et al (15) demonstrated that there was no significant difference in the mean range of motion (ROM) or Knee Society score (KSS) between MB and FB implants, at a mean follow-up of 13.4 months. However, short-term results are not always indicative of mid- and long-term outcomes. Controversy exists with respect to the clinical and radiological differences in outcomes between FB and MB TKAs during the mid- to long-term follow-up. Matsuda et al (17) revealed that no differences were exhibited in the rotational alignment or the ROM between MB and FB implants for a total of 61 knee arthroplasties in the mid-term follow-up. By contrast, a study by Higuchi et al (18), of $76 \mathrm{TKAs}$, demonstrated that the postoperative-extension ROM was significantly improved following TKA using an MB implant, in comparison with that employing an FB implant, at the four-year follow-up.

The mid- to long-term results of the prosthesis are important in evaluating the efficiency of implant. Although several meta-analyses concerning the clinical differences between FB and MB TKAs have been performed (19-22), the pooled data were not analyzed according to the different follow-up periods. In addition, a number of new randomized controlled trials (RCTs) have recently emerged. Therefore, there is a requirement for a new systematic review to compare the clinical, radiological and general health results of the two types of prostheses, at the mid- to long-term follow-up period.

\section{Methods}

Search strategy. We searched the Cochrane Central Register of Controlled Trials (CENTRAL) in the Cochrane Library, Medline and Embase. The search strategies used are demon- 
Table I. Search strategies.

\begin{tabular}{lll}
\hline Database & Period of search & Search strategy
\end{tabular}

Medline (Pubmed)

1966-August 2012
Cochrane Central Register of

Controlled Trials in the

Cochrane library

Issue 2, 2012

(ssue 2,2012

Cochrane library

Embase

1974-August 2012
(((((“Arthroplasty, Replacement, Knee”[Mesh])) OR (((knee))

AND ((()(replacement*) OR arthroplast*) OR prosthe*) OR implant) OR endoprosthe* $))))$ AND $(((($ (mobile bearing) OR mobile platform) OR rotating platform) OR meniscal bearing) OR gliding bearing)) AND ((randomized controlled trial[pt] OR controlled clinical trial[pt] OR randomized[tiab] OR placebo [tiab] OR clinical trials as topic[mesh:noexp] OR randomly [tiab] OR trial[ti]) NOT (animals[mh]NOT humans[mh]))

\#1 MeSH descriptor Arthroplasty, Replacement, Knee explode all trees

\#2 (replacement*) or (arthroplast*) or (prosthe*) or (implant) or (endoprosthe*)

\#3 (knee)

\#4 (\#2 AND \#3)

\#5 (\#1 AND \#4)

\#6 (mobile bearing) or (mobile platform) or (rotating platform) or (meniscal bearing) or (gliding bearing)

\#7 (\#1 AND \#6)

'knee arthroplasty'/exp OR 'total knee replacement'/exp OR (replacement* OR arthroplast* OR prosthe* OR implant OR endoprosthe* AND knee) AND (mobile AND bearing OR (mobile AND platform) OR (rotating AND platform) OR (meniscal AND bearing) OR (gliding AND bearing)) AND (random* OR blind* OR placebo OR 'meta analysis') strated in Table I, along with the time span for the searches. The bibliography of eligible studies was also searched to identify further relevant trials.

Eligibility criteria. The inclusion criteria for the articles selected were as follows: i) the studies involved adult patients who had undergone primary TKA; ii) the studies were RCTs comparing MB- and FB TKAs and iii) the mean follow-up was $>5$ years. Animal and cadaver studies were excluded, and no language restriction was used.

Data extraction. Data were extracted independently from the included studies by two reviewers (MC/DC), and disagreements were resolved through consensus. Relevant data included demographic information, methodological quality, duration of follow-up, clinical and radiographical outcomes, patient preferences and complications. Whenever studies pertained to the same population at different follow-up periods, the investigation with the longer duration of follow-up was retained to avoid the duplication of information. Authors were contacted to request any unclear or missing data. Disagreements concerning paper eligibility were resolved through discussion.

Assessment of methodological quality. The methodological quality of the trials was evaluated independently by two reviewers $(\mathrm{MC} / \mathrm{DC})$, without masking the trial names. The reviewers followed the instructions provided in the Cochrane Handbook for Systematic Reviews of Interventions (23). The following domains were assessed: sequence generation, allocation concealment, blinding, incomplete data outcomes, revealing of selective outcomes and any remaining biases. When the information in the study was inadequate, attempts were made to contact the authors in order to ensure that the study was evaluated correctly.

Data analysis. The meta-analysis was conducted in accordance with the recommendations of the Cochrane Collaboration, using Review Manager (RevMan) software, version 5 (Copenhagen: The Nordic Cochrane Centre, The Cochrane Collaboration, 2008). For continuous outcomes, the weighted mean difference (WMD) and the $95 \%$ confidence interval (CI) were calculated. For dichotomous outcomes, the relative ratio (RR) and the $95 \% \mathrm{CI}$ were calculated. Heterogeneity was explored using the $\chi^{2}$ test and the $\mathrm{I}^{2}$ statistic. Heterogeneity was considered to be significant when the P-value from the $\chi^{2}$ test was $<0.10$, or when the $\mathrm{I}^{2}$ statistic was $>50 \%$. A fixed-effect model was used if there was no statistical evidence of heterogeneity; otherwise, a random-effect model was selected. The analysis was conducted on an intention-to-treat bias, whenever possible, and a funnel plot was used to explore the publication bias. $\mathrm{P}<0.05$ was considered to indicate a statistically significant difference. 


\section{Results}

Description of studies. A preferred reporting items for systematic reviews and meta-analyses (PRISMA) flow diagram outlining the literature search results is summarized in Fig. 1. In the initial search, a total of 220 references were identified electronically (Embase, 75; Medline, 92 and CENTRAL, 53). Following the application of the inclusion and exclusion criteria to the study titles and abstracts, only nine trials fulfilled the inclusion criteria.

Risk of bias in included studies. The risk of bias is demonstrated graphically (Fig. 2) and also summarized (Fig. 3). Six (24-29) of the nine trials $(66.7 \%)$ exhibited adequate sequence generation in the randomization process. However, only two trials $(24,25)$ stated the method of allocation concealment. In the study by Lädermann et al (25), the allocation concealment was performed using sealed opaque envelopes, which were opened within $24 \mathrm{~h}$ prior to surgery; whereas in the study by Mahoney et al (24), allocation concealment was ensured by centralized randomization. Four of the nine trials $(24-26,29)$ performed patient blinding. Clinician blinding of the implant type was impossible. All studies demonstrated assessor blinding, with the exception of two trials $(24,30)$. A funnel plot analysis of the combined trials indicated a symmetry, demonstrating minimal recording bias (Fig. 4).

\section{Effects of intervention}

Knee score. The KSS (25,26,29,31), the Hospital for Special Surgery (HSS) knee score $(30,31)$ and the Western Ontario and McMaster Universities Osteoarthritis Index (WOMAC) (26) were used for the clinical assessment of the patients (in four, two and one trials, respectively). The meta-analysis revealed no significant difference in the KSS between the FB and $\mathrm{MB}$ groups $(\mathrm{WMD}=0.80 ; 95 \% \mathrm{CI},-1.36-2.97 ; \mathrm{P}=0.47$ ), and heterogeneity tests indicated minimal interstudy heterogeneity $\left(\mathrm{P}=0.98\right.$ and $\mathrm{I}^{2}=0 \%$; fixed-effect analysis; Fig. 5). In addition, a fixed-effect model of the pooled data demonstrated that there was no statistical difference in the HSS knee score between the two groups (WMD=-0.65; 95\% CI, -3.07-1.77; $\mathrm{P}=0.60$ ). The study conducted by Jolles et al (26) was the sole study to assess WOMAC scores, and revealed that there was no significant difference between the groups $(\mathrm{P}=0.94)$.

Four of the trials stated the KSS-function outcomes $(25-27,29)$. There was no indication of statistical heterogeneity between the trials $\left(\mathrm{P}=0.52\right.$ and $\left.\mathrm{I}^{2}=0 \%\right)$, and a fixed-effect model of the pooled data revealed no significant difference in the KSS-function outcomes between the FB and MB TKAs (WMD=-0.16; 95\% CI, -3.60-3.27; P=0.93; Fig. 6).

Maximum knee flexion. There were three trials (551 knees) that stated the maximum knee flexion $(25,27,31)$. The meta-analysis did not demonstrate a significant difference between the two groups (WMD=-0.52; 95\% CI, -3.36-2.32; $\mathrm{P}=0.72)$, and there was minimal interstudy heterogeneity $\left(\mathrm{P}=0.47\right.$ and $\mathrm{I}^{2}=0 \%$; fixed-effect analysis).

Pain. The meta-analysis of the visual analog scales (VAS) of pain in two of the trials indicated no significant difference between the MB and FB TKAs (WMD=-0.02; $95 \% \mathrm{CI}$, -0.62-0.59; $\mathrm{P}=0.96)$, with minimal interstudy heterogeneity $\left(\mathrm{P}=0.66\right.$ and $\mathrm{I}^{2}=0 \%$; fixed-effect analysis $)(25,26)$.

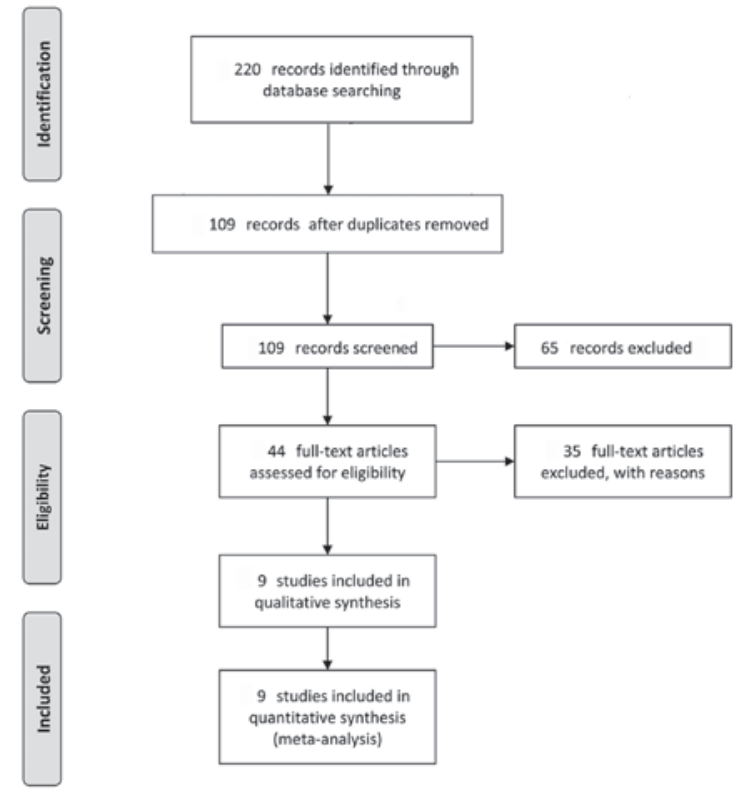

Figure 1. Preferred reporting items for systematic reviews and meta-analyses (PRISMA) flow diagram outlining literature search results.

Patient preference. Only one trial revealed the patients' preferences with regard to their knee replacements. In the study by Matsuda et al (17), 13 patients underwent bilateral arthroplasty, where one knee was replaced with an FB prosthesis and the other with an MB prosthesis. In these patients, one patient favored the knee with the FB prosthesis, two patients favored the $\mathrm{MB}$ prosthesis and the remaining ten patients stated that there was no difference between their knees.

Quality of life. Three methods were used to measure the quality of life in the selected studies. Shemshaki et al (29) evaluated short form 36 (SF-36) scores, Lädermann et al (25) assessed SF-12 scores and Jolles et al (26) used EuroQol-5D scores. No significant differences in the quality of life scores between the MB and FB TKAs were demonstrated in the studies $(\mathrm{P}>0.05)$.

Radiological outcomes. There were two trials (277 knees) that revealed the occurrence of nonprogressive radiolucent lines around the tibial or femoral components $(26,27)$. The meta-analysis demonstrated that there was no significant difference between the FB and MB TKAs ( $\mathrm{RR}=1.05 ; 95 \%$ CI, 0.50-2.18; $\mathrm{P}=0.90$ ).

Survivorship. Three studies $(24,27,28)$ revealed the Kaplan-Meier survival estimates for the prostheses; however, two of these studies $(24,28)$ did not contain sufficient information for the meta-analysis. Kalisvaart et al (27) indicated that no difference was detected in implant durability or fixation between the FB and MB prostheses after five years; Kaplan-Meier analysis demonstrated five-year implant survival rates of 98.0 and $98.7 \%$ for the FB and MB groups, respectively, with revision surgery for any reason taken as the end point. Kim et al (28) reported that the Kaplan-Meier survival estimate revealed 99 and $100 \%$ survival rates of the prosthesis in the $\mathrm{MB}$ and $\mathrm{FB}$ groups, respectively, after five years (95\% CI, 0.89-0.97), with all reasons for failure taken into account. Mahoney et al (24) indicated that survival was similar $(\mathrm{P}=0.351)$ between the MB and FB groups, with revi- 


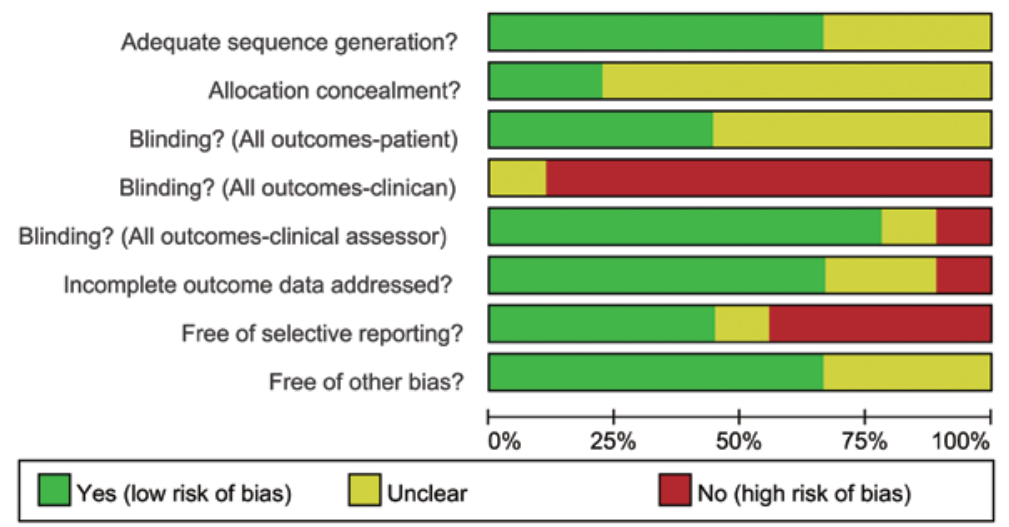

Figure 2. Risk of bias graph: a review of the authors' judgments regarding each risk of bias item, presented as percentages across all included studies.

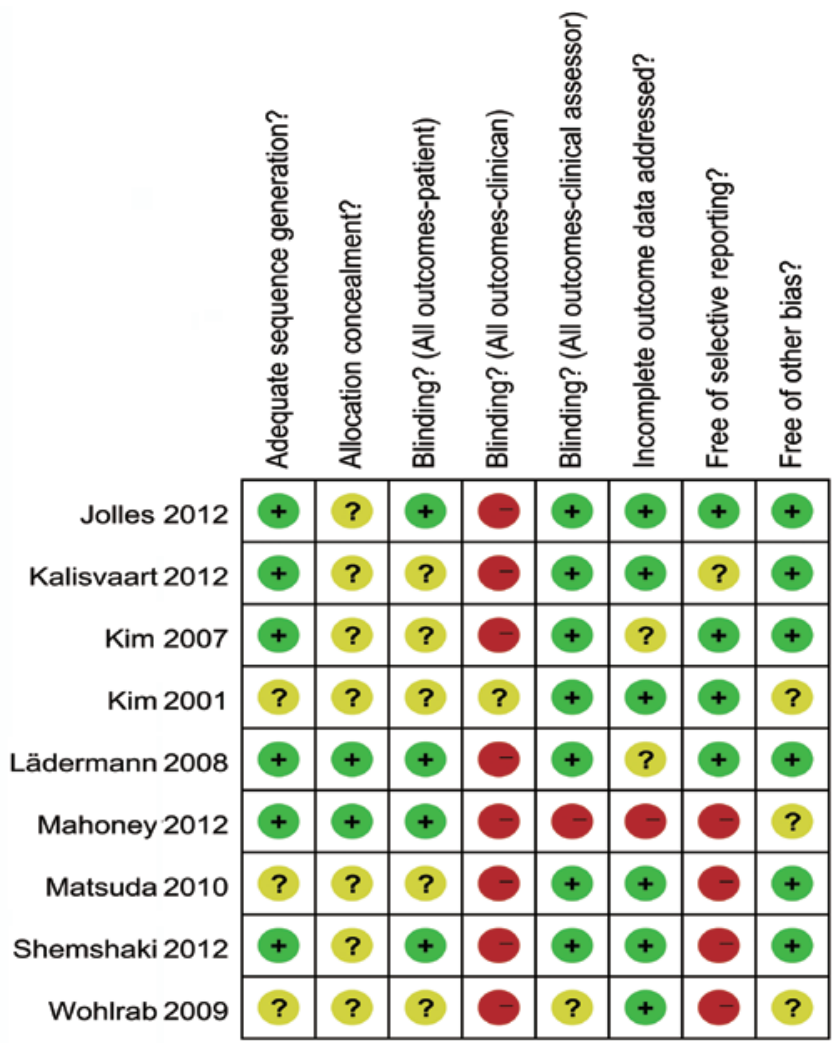

Figure 3. Risk of bias summary: a review of the authors' judgments regarding each risk of bias item, for each included study.

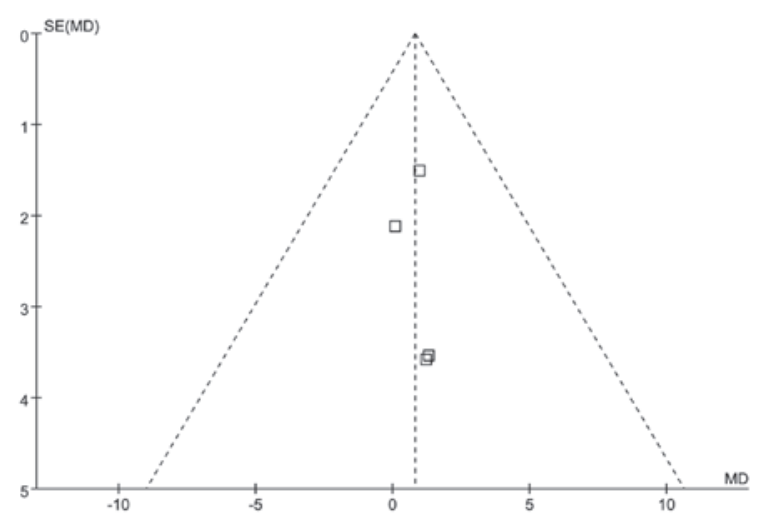

Figure 4. Funnel plot for the studies that utilized the Knee Society score (KSS). sion of any component for any reason taken as the end point [90.1\% (95\% CI, 84.1-93.9) and 94.2\% (95\% CI, 90.1-96.6) for the MB and FB groups, respectively).

Complications. Five of the studies provided data on postoperative complications, including aseptic loosening, infection, revision, patellar crepitus, complete wear of the polyethylene tibial bearing and knee stiffness $(19,20,22,23,27)$. The meta-analysis did not identify any significant differences in the RRs between MB and FB TKAs, with respect to aseptic loosening, infection, revision, patellar crepitus or knee stiffness (Table II). Kim et al (31) reported two cases of complete wear of the polyethylene tibial bearing in the FB cohort, and one case of dislocation of the medial polyethylene tibial bearing in the MB cohort. 
Table II. Meta-analysis of the complications in fixed-bearing and mobile-bearing TKAs.

\begin{tabular}{|c|c|c|c|c|c|c|c|}
\hline \multirow[b]{2}{*}{ Complication } & \multirow[b]{2}{*}{ Studies assessed } & \multicolumn{2}{|c|}{ Incidence } & \multirow{2}{*}{$\begin{array}{l}\text { Relative Risk } \\
\quad(95 \% \mathrm{CI})\end{array}$} & \multirow{2}{*}{$\begin{array}{c}\text { Overall effect } \\
\text { (P-value) }\end{array}$} & \multicolumn{2}{|c|}{ Heterogeneity } \\
\hline & & Mobile & Fixed & & & $\mathrm{I}^{2}(\%)$ & $\chi^{2}(\mathrm{P}$-value $)$ \\
\hline Aseptic loosening & $(19,22)$ & $2 / 328$ & $3 / 406$ & $1.11(0.21,5.89)$ & 0.90 & 44 & 0.18 \\
\hline Revision & $(19,20,27)$ & $25 / 412$ & $17 / 419$ & $1.48(0.82,2.67)$ & 0.20 & 0 & 0.60 \\
\hline Infection & $(19,20,22,27)$ & $7 / 546$ & $2 / 628$ & $2.75(0.81,9.33)$ & 0.11 & 0 & 0.85 \\
\hline Patellar crepitus & $(22,23)$ & $45 / 250$ & $53 / 325$ & $0.86(0.61,1.21)$ & 0.39 & 0 & 0.55 \\
\hline Knee stiffness & $(19,20,22)$ & $9 / 372$ & $7 / 454$ & $1.56(0.62,3.94)$ & 0.35 & 0 & 0.55 \\
\hline
\end{tabular}

TKA, total knee arthroplasty; CI, confidence interval.

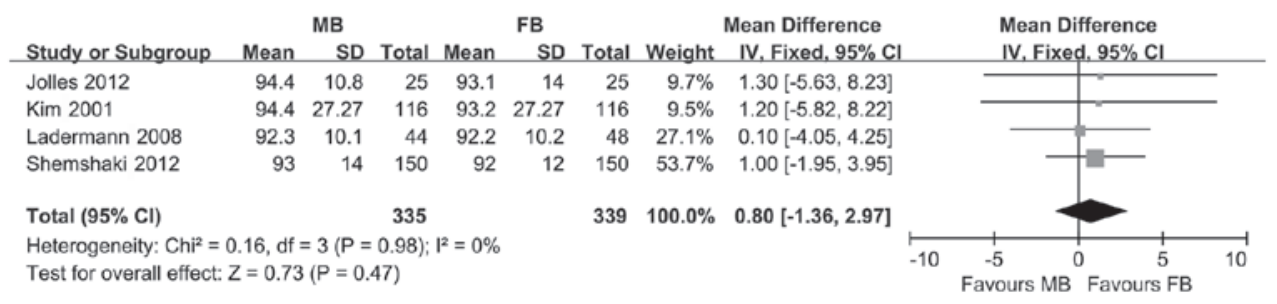

Figure 5. Forest plot comparing the Knee Society scores of mobile-bearing (MB) and fixed-bearing (FB) implants. CI, confidence interval.

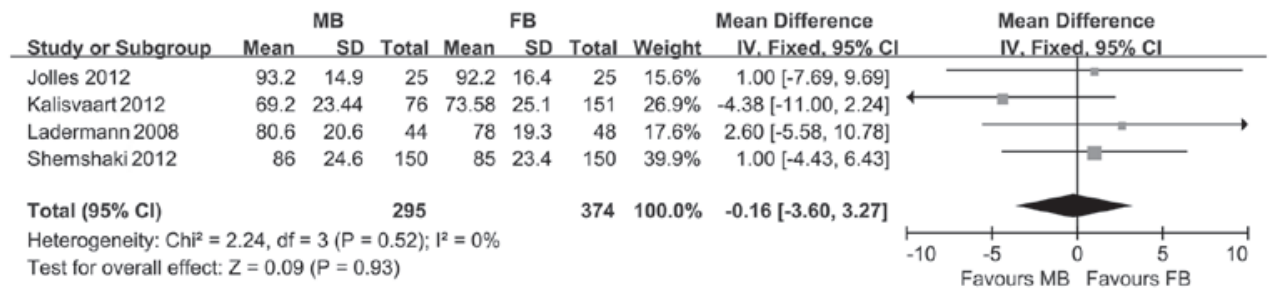

Figure 6. Forest plot comparing the Knee Society functional outcome scores of mobile-bearing (MB) and fixed-bearing (FB) implants. CI, confidence interval.

\section{Discussion}

There has been a great interest in MB prostheses, due to their assumed reduction in polyethylene wear and component loosening, in comparison with FB prostheses (32-34). The current study was designed to determine whether this theoretical superiority has been translated into improved clinical outcomes at the mid- to long-term follow-up. The results of this review demonstrated that there was no significant difference with respect to clinical, functional or radiological outcomes, or complication rates between the FB and MB TKA designs. However, the review has revealed two factors to be considered.

The mean follow-up duration of the trials included in this study was 5 years, and only two trials $(25,31)$ had a follow-up duration of $>7$ years. The expected advantages of the MB prosthesis, i.e. reduced wear and diminished implant loosening, may only become apparent following a long period of time. Therefore, trials with a longer follow-up duration are required before a definite conclusion may be reached. In addition, different MB implant designs were used in different trials. Although all the designs featured polyethylene-bearing mobility, the MB implants differed according to whether the posterior cruciate ligament was retained or sacrificed, or whether the implant was substituted with a different brand. It has been demonstrated that different types of implant elicit different effects on the ROM achieved following the TKA (35). It is possible that the variability amongst the different $\mathrm{MB}$ and FB prostheses may have adversely impacted the accuracy of the conclusion of the present study.

Polyethylene wear was a predominant reason for the development of MB designs. The aim of the MB design is to reduce the overall polyethylene wear through increased contact area and congruency, while minimizing the constraint and maintaining the normal knee motion. A study conducted by Parks et al (36) demonstrated that an undersurface stress existed between the MB undersurface of the polyethylene and the metal tray that was $40 \%$ of the uppersurface stress, and retrieval studies indicated that the MB inserts had an improved wear rate in comparison with the FB inserts (0.04 versus $0.07 \mathrm{~mm} /$ year, respectively) (37). However, Kelly et al (38) demonstrated that the MB surface was damaged to an extent similar to that of the FB surface, in a study of 48 retrieved MB TKAs. Kim et al (31) revealed that there were two cases that were revised due to complete wear of the polyethylene tibial 
bearing in the FB cohort; however, no wear rate was revealed in any of the trials included in the present study.

As mentioned previously, the MB TKAs may theoretically prolong the implant survival by reducing shear forces at the polyethylene interface, reducing contact forces and enabling the mobility of the bearing surface (39-41). However, in the current review, the three studies that revealed the Kaplan-Meier survival estimates did not demonstrate any significant differences between the MB and FB TKAs at the five-year follow-up. Certain studies revealed the survivorship for TKA as $91 \%$ at 14 years (42) and $92 \%$ at 15 years (43), with repeated surgery for any reason as the endpoint. Therefore, a sufficiently long-term follow-up is required in order to evaluate the survivorship of TKA.

Allocation concealment is one of the most important biases to minimize when establishing the quality of the evidence. Since only two of the nine trials clearly described the method of allocation concealment used, it is possible that allocation bias occurred in the other trials. The blinding of patients was accomplished in $<50 \%$ of trials, and the evidence was indeterminate, as it related to functional assessment. However, the blinding of the clinical assessors was performed in the majority of the studies, which reduced the detection bias. Certain trials did not reveal important results, which diminished the ability of the present study to estimate the effectiveness of the treatments. The interpretation of the results of the studies was limited, as, according to the criteria for the judgement of the risk of bias, all the studies were at a high risk of bias.

In conclusion, the results of this meta-analysis suggest that $\mathrm{MB}$ and FB prostheses have no statistically significant differences in clinical or radiological outcomes, or in complication rates. In order to perform an improved assessment of the efficacy of MB implants, well-designed RCTs that compare FB and MB prostheses, and have a long-term follow-up, are required.

\section{References}

1. Gandhi R, Dhotar H, Razak F, Tso P, Davey JR and Mahomed NN Predicting the longer term outcomes of total knee arthroplasty. Knee 17: 15-18, 2010.

2. Gioe TJ, Sinner P, Mehle S, Ma W and Killeen KK: Excellent survival of all-polyethylene tibial components in a community joint registry. Clin Orthop Relat Res 464: 88-92, 2007.

3. Ritter MA: The Anatomical Graduated Component total knee replacement: a long-term evaluation with 20-year survival analysis. J Bone Joint Surg Br 91: 745-749, 2009.

4. Robertsson O, Bizjajeva S, Fenstad AM, et al: Knee arthroplasty in Denmark, Norway and Sweden. Acta Orthop 81: 82-89, 2010.

5. Spencer SJ, Baird K, Young D and Tait GR: The Rotaglide mobile bearing knee arthroplasty: A 10- to 13-year review from an independent centre. Knee 19: 20-23, 2012.

6. Bottlang M, Erne OK, Lacatusu E, Sommers MB and Kessler O: A mobile-bearing knee prosthesis can reduce strain at the proximal tibia. Clin Orthop Relat Res 447: 105-111, 2006.

7. Dennis DA and Komistek RD: Kinematics of mobile-bearing total knee arthroplasty. Instr Course Lect 54: 207-220, 2005.

8. Stukenborg-Colsman C, Ostermeier S, Hurschler C and Wirth CJ: Tibiofemoral contact stress after total knee arthroplasty: comparison of fixed and mobile-bearing inlay designs. Acta Orthop Scand 73: 638-646, 2002.

9. Li ZJ, Zhang K and Kim TK: Mobile- and fixed-bearing total knee arthroplasty for knee osteoarthritis: Comparisons of early clinical outcomes. Journal of Clinical Rehabilitative Tissue Engineering Research 12: 9589-9593, 2008 (In Chinese).

10. Callaghan JJ, Insall JN, Greenwald AS, et al: Mobile-bearing knee replacement: concepts and results. Instr Course Lect 50: 431-449, 2001.
11. Jacobs WC, Christen B, Wymenga AB, et al: Functional performance of mobile versus fixed bearing total knee prostheses: a randomised controlled trial. Knee Surg Sports Traumatol Arthrosc 20: 1450-1455, 2012.

12. Holinka J, Bahrami N, Lass R, Frantal S, Windhager R and Wanivenhaus HA: No difference in ROM and knee function between mobile and floating platforms in TKA. Knee Surg Sports Traumatol Arthrosc: Jun 29, 2012 (Epub ahead of print).

13. Lampe F, Sufi-Siavach A, Bohlen KE, Hille E and Dries SP: One year after navigated total knee replacement, no clinically relevant difference found between fixed bearing and mobile bearing knee replacement in a double-blind randomized controlled trial. Open Orthop J 5: 201-208, 2011.

14. Ball ST, Sanchez HB, Mahoney OM and Schmalzried TP: Fixed versus rotating platform total knee arthroplasty: a prospective, randomized, single-blind study. J Arthroplasty 26: 531-536, 2011.

15. Hanusch B, Lou TN, Warriner G, Hui A and Gregg P: Functional outcome of PFC Sigma fixed and rotating-platform total knee arthroplasty. A prospective randomised controlled trial. Int Orthop 34: 349-354, 2010.

16. Harrington MA, Hopkinson WJ, Hsu P and Manion L: Fixedvs mobile-bearing total knee arthroplasty: does it make a difference? - a prospective randomized study. J Arthroplasty 24: 24-27, 2009.

17. Matsuda S, Mizu-uchi H, Fukagawa S, et al: Mobile-bearing prosthesis did not improve mid-term clinical results of total knee arthroplasty. Knee Surg Sports Traumatol Arthrosc 18: 1311-1316, 2010.

18. Higuchi H, Hatayama K, Shimizu M, Kobayashi A, Kobayashi T and Takagishi K: Relationship between joint gap difference and range of motion in total knee arthroplasty: a prospective randomised study between different platforms. Int Orthop 33: 997-1000, 2009.

19. Wen Y, Liu D, Huang Y and Li B: A meta-analysis of the fixed-bearing and mobile-bearing prostheses in total knee arthroplasty. Arch Orthop Trauma Surg 131: 1341-1350, 2011.

20. Carothers JT, Kim RH, Dennis DA and Southworth C: Mobile-bearing total knee arthroplasty: a meta-analysis. J Arthroplasty 26: 537-542, 2011.

21. Smith TO, Ejtehadi F, Nichols R, Davies L, Donell ST and Hing CB: Clinical and radiological outcomes of fixed- versus mobile-bearing total knee replacement: a meta-analysis. Knee Surg Sports Traumatol Arthrosc 18: 325-340, 2010.

22. Oh KJ, Pandher DS, Lee SH, Sung Joon SD Jr and Lee ST: Meta-analysis comparing outcomes of fixed-bearing and mobile-bearing prostheses in total knee arthroplasty. J Arthroplasty 24: 873-884, 2009.

23. Higgins JPT and Green S (eds): Cochrane Handbook for Systematic Reviews of Interventions. Version 5.1.0 [updated March 2011]. The Cochrane Collaboration, 2011. Available from www.cochrane-handbook.org.

24. Mahoney OM, Kinsey TL, D'Errico TJ and Shen J: The John Insall Award: no functional advantage of a mobile bearing posterior stabilized TKA. Clin Orthop Relat Res 470: 33-44, 2012.

25. Lädermann A, Lübbeke A, Stern R, Riand N and Fritschy D: Fixed-bearing versus mobile-bearing total knee arthroplasty: a prospective randomised, clinical and radiological study with mid-term results at 7 years. Knee 15: 206-210, 2008.

26. Jolles BM, Grzesiak A, Eudier A, et al: A randomised controlled clinical trial and gait analysis of fixed- and mobile-bearing total knee replacements with a five-year follow-up. J Bone Joint Surg Br 94: 648-655, 2012.

27. Kalisvaart MM, Pagnano MW, Trousdale RT, Stuart MJ and Hanssen AD: Randomized clinical trial of rotating-platform and fixed-bearing total knee arthroplasty: no clinically detectable differences at five years. J Bone Joint Surg Am 94: 481-489, 2012.

28. Kim YH, Kim DY and Kim JS: Simultaneous mobile- and fixed-bearing total knee replacement in the same patients. A prospective comparison of mid-term outcomes using a similar design of prosthesis. J Bone Joint Surg Br 89: 904-910, 2007.

29. Shemshaki H, Dehghani M, Eshaghi MA and Esfahani MF: Fixed versus mobile weight-bearing prosthesis in total knee arthroplasty. Knee Surg Sports Traumatol Arthrosc 20: 2519-2527, 2012

30. Wohlrab D, Hube R, Zeh A and Hein W: Clinical and radiological results of high flex total knee arthroplasty: a 5 year follow-up. Arch Orthop Trauma Surg 129: 21-24, 2009.

31. Kim YH, Kook HK and Kim JS: Comparison of fixed-bearing and mobile-bearing total knee arthroplasties. Clin Orthop Relat Res 392: 101-115, 2001. 
32. Buechel FF Sr: Long-term followup after mobile-bearing total knee replacement. Clin Orthop Relat Res 404: 40-50, 2002

33. Sorrells RB, Stiehl JB and Voorhorst PE: Midterm results of mobile-bearing total knee arthroplasty in patients younger than 65 years. Clin Orthop Relat Res 390: 182-189, 2001.

34. Buechel FF: Mobile-bearing knee arthroplasty: rotation is our salvation! J Arthroplasty 19 (Suppl 1): 27-30, 2004.

35. Jacobs WC, Clement DJ and Wymenga AB: Retention versus sacrifice of the posterior cruciate ligament in total knee replacement for treatment of osteoarthritis and rheumatoid arthritis. Cochrane Database Syst Rev: CD004803, 2005.

36. Parks NL, Engh GA, Topoleski LD and Emperado J: The Coventry Award. Modular tibial insert micromotion. A concern with contemporary knee implants. Clin Orthop Relat Res 356: 10-15, 1998.

37. Berry DJ, Currier JH, Mayor MB and Collier JP: Knee wear measured in retrievals: a polished tray reduces insert wear. Clin Orthop Relat Res 470: 1860-1868, 2012.
38. Kelly NH, Fu RH, Wright TM and Padgett DE: Wear damage in mobile-bearing TKA is as severe as that in fixed-bearing TKA. Clin Orthop Relat Res 469: 123-130, 2011.

39. Schunck J and Jerosch J: Knee arthroplasty. Mobile- and fixed-bearing design. Orthopade 32: 477-483, 2003 (In German).

40. Chouteau J, Lerat JL, Testa R, Moyen B, Fessy MH and Banks SA: Mobile-bearing insert translational and rotational kinematics in a PCL-retaining total knee arthroplasty. Orthop Traumatol Surg Res 95: 254-259, 2009.

41. Vogt JC and Saarbach C: LCS mobile-bearing total knee replacement. A 10-year's follow-up study. Orthop Traumatol Surg Res 95: 177-182, 2009.

42. Parsch D, Krüger M, Moser MT and Geiger F: Follow-up of 11-16 years after modular fixed-bearing TKA. Int Orthop 33: 431-435, 2009.

43. Roberts VI, Esler CN and Harper WM: A 15-year follow-up study of 4606 primary total knee replacements. J Bone Joint Surg Br 89: 1452-1456, 2007. 